\title{
关于 Köthe 问题及幂零性问题
}

许 永 华

(复旦大学数学研究所, 上海)

\section{畝要}

本文的主要结果有二: 一是给出一个环成立 Köthe 猜测的充要条件; 另一是给 出环的任一诣零单侧理想必是幕零的充要条件.

本文所讨论的环都是结合环. 先研究关于 Köthe 猜测: 环 $R$ 的任一非零诣零左理想所生 成的理想是诣零的. 这个著名问题直到现在未曾解决, 本文首先引进 Köthe 子集及 $R$-左模 同态链归纳条件的概念，并获得了如下的结果：环 $R$ 成立 Köthe 猜测当且仅当 $R$ 满足每个 Köthe 子集上 $R$-左模同态链归纳条件. 值得指出的是, $R$-左模同态链归纳条件已不是一种 有限条件，因此它对环的结构研究具有独立的兴趣,但诸如左零化子极大条件及左零因子理想 极大条件等等有限条件,都满足 $R$-左模同态链归纳条件.

本文另一个研究问题是: 环 $R$ 的任一诣零单侧理想是幕零的问题. 自从 Herstein ${ }^{[1]}$ 猜测 被否定以来，人们继续寻求充分条件使任一诣零单侧理想是幂零的. 最近谢邦杰 ${ }^{[2]}$ 提出左零 因子理想极大条件, 它能使上述结论成立. 本文第二节获得了一个环 $R$ 的每个诣零单侧理想 是幕零的充要条件，我们的定理是：设 $R$ 是环, $N^{\prime}$ 是 $R$ 的诣罢根， $N$ 是 $R$ 的所有幕零理想之 和,记 $M=N^{\prime}-N$, 那末 $R$ 的每个诣零单侧理想是幕岺的当且仅当 $R$ 在 $M$ 上及每个 Köthe: 子集上满足 $R$-左模同态链归纳条件,并且 $N$ 是幕零的.

由此定理我们立刻得出如下结论: 设 $R$ 是环，若 $R$ 满足左琴化子极大条件，且 $N$ 是幂零 的, 那末 $R$ 的任一诣零单侧理想是幂零的.

由此推论可见, Herstein 猜测被否定, 其原因是没有假设 $N$ 是幂零的. 因此仅仅左零化子 极大条件不能得出 $N$ 是幂零的.

另一方面, 谢邦杰的左零因子极大条件, 必使 $N$ 是幂零的, 并且必使左零化子极大条件成 立. 因此我们定理自然包含谢邦杰的结果.

本节研究 Köthe 问题. 我们将给出一个环 $R$ 成立 Köthe 猜测的充要条件.

在讨论 Köthe 问题之前, 我们先来叙述一下概念：设 $R$ 是环, $S$ 是 $R$ 的一个子集, 记 ${ }^{\perp} S=$ $\{r \in R \mid r S=0\}$, 并称 ${ }^{\perp} S$ 是 $R$ 的一个左零化子. 特别 $S$ 只含一个元素 $x$, 那末称 ${ }^{{ }^{\prime} x}=\{r \in R$ $\mid r x=0\}$ 为 $R$ 的一个特殊左零化子. 
定义 1.1. 设 $R$ 是一个环， $M$ 是 $R$ 的一个子集，我们说 $R$ 在 $M$ 上满足特殊左零化子极大条 件, 若对于任一个特殊左零化子升链 ${ }^{+} x_{1} \subseteq{ }^{\perp} x_{2} \subseteq \cdots$, 其中 $x_{i} \in M$, 必存在正整数 $n$, 使 ${ }^{\perp} x_{n}=$ $\perp_{x_{n+1}}=\cdots$.

如果 $M$ 取作整个环 $R$ 时,我们就简称 $R$ 满足特殊左零化子极大条件.

根据定义 1.1 易知: 满足通常左零化子极大条件 ${ }^{1)}$ 的环必满足特殊左零化子极大条件. 反 之,显然一般不成立. 因此,定义 1.1 的条件大大减弱通常左零化子条件.

但是定义 1.1 的 $M$ 上特殊左零化子极大条件,还是有限链条件的一种形式.下面我们要引 进 $R$-左模同态链归纳条件的概念, 它不仅减弱定义 1.1 的 $M$ 上特殊左零化子极大条件, 更重要 的是它已脱离有限链条件概念, 即它一般不是有限条件形式的链, 因此它本身及其对扩大环类 结构的研究具有独立的兴趣.

定义 1.2. 设 $R$ 是一个环, $M$ 是 $R$ 的一个子集,我们称 $R$ 的一个左理想集合 $\left\{R x_{i}\right\}_{l}$ 为 $M$ 上 $R$-左模同态链当且仅当 i) 所有 $x_{i} \in M, i \in I$, ii) 令 $R x_{\alpha}, R x_{\beta}$ 是 $\left\{R x_{i}\right\}_{i \in I}$ 中任二元素, 那 末或者存在 $R x_{\beta}$ 到 $R x_{\alpha}$ 上的模同态映照 $\varphi_{\beta, \alpha}$, 使 $x_{\beta} \varphi_{\beta, \alpha}=x_{\alpha}$, 或者存在 $R x_{\alpha}$ 到 $R x_{\beta}$ 上 的模同态映照 $\varphi_{\alpha, \beta}$, 使 $x_{\alpha} \varphi_{\alpha, \beta}=x_{\beta}$.

定义 1.3. 一个 $M$ 上 $R$-左模同态链 $\left\{R x_{i}\right\}_{i \in I}$ 称为归纳的, 若存在一个 $R$-左模 $R x, x \in M$, 对于 $\left\{R x_{i}\right\}_{i \in I}$ 中任一元素 $R x_{\alpha}$ 必存在模同态 $\varphi_{\alpha}$, 使 $R x_{\alpha}$ 映到 $R x$ 上,且 $x_{\alpha} \varphi_{\alpha}=x$ 。如果 有另一左模 $R x^{\prime}, x^{\prime} \in M$, 对于 $\left\{R x_{i}\right\}_{i \in I}$ 中任一元素 $R x_{\beta}$ 存在一个从 $R x_{\beta}$ 到 $R x^{\prime}$ 上的模 同态 $\varphi_{\beta}^{\prime}$, 使 $x_{\beta} \varphi_{\beta}^{\prime}=x^{\prime}$, 那末必有模同态 $\varphi$, 使 $R x$ 映到 $R x^{\prime}$ 上,且 $x \varphi=x^{\prime}$.

定义 1.4. 我们说环 $R$ 满足 $M$ 上 $R$-左模同态链归纳条件,当且仅当 $R$ 的任一 $M$ 上 $R$-左模 同态链皆是归纳的.

性质 1.1. 设 $R$ 是满足 $M$ 上特殊左零化子极大条件, 那末 $R$ 必满足 $M$ 上 $R$-左模同态链归 纳条件.

证 记 $\left\{R x_{i}\right\}_{i \in I}$ 是 $M$ 上任一 $R$-左模同态链, $R x_{\alpha}$ 及 $R x_{\beta}$ 是 $\left\{R x_{i}\right\}_{i \in I}$ 的任二元素, $\varphi_{\alpha, \beta}$ 是 $R x_{\alpha}$ 到 $R x_{\beta}$ 上的一个模同态, 且 $x_{\alpha} \varphi_{\alpha, \beta}=x_{\beta}$, 那末易知 ${ }^{\perp} x_{\beta} \supseteq{ }^{\perp} x_{\alpha}$, 因此 $\left\{{ }^{-} x_{\beta}\right\}_{\beta \in I}$ 即是通常包 含关系意义下的一个链. 由于 $R$ 满足 $M$ 上特殊左零化子极大条件, 因此必有元素 ${ }^{\perp} x \in\left\{{ }^{\perp} x_{\beta}\right\}_{\beta \in I}$ 使所有 ${ }^{{ }^{\prime}} x_{\beta} \subseteq{ }^{\perp} x, \beta \in I$. 这就是说, 对于 $\left\{R x_{i}\right\}_{i \in I}$ 的任一元素 $R x_{\beta}$ 必存在模同态 $\varphi_{\beta}$, 使 $R x_{\beta}$ 映到 $R x$ 上,且 $x_{\beta} \varphi_{\beta}=x$. 另一方面, 如果存在另一个左模 $R x^{\prime}, x^{\prime} \in M$, 对于 $\left\{R x_{i}\right\}_{i \in l}$ 中 任一元素 $R x_{\beta}$ 有一个模同态 $\varphi_{\beta}^{\prime}$, 使 $R x_{\beta}$ 映到 $R x^{\prime}$ 上,且 $x_{\beta} \varphi_{\beta}^{\prime}=x^{\prime}$, 那末特别取 $x_{\beta}=x$ 时, 就有前面所述 $\varphi_{\beta}^{\prime}$ ，使 $x \varphi_{\beta}^{\prime}=x^{\prime}$ ，因此，由定义 1.3 知， $\left\{R x_{i}\right\}_{i \in I}$ 是归纳的.

推论 1.1. 设 $R$ 是满足通常左零化子极大条件, 则 $R$ 必满足 $R$-左模同态链归纳条件.

引理 1.1. 设 $R$ 是结合环. 那末任何诣零左理想必含在一个极大诣零左理想之中.

证. 设 $L$ 是一个诣零左理想，记 $S$ 是所有含 $L$ 的诣零左理想全体. 若 $L_{1} \subseteq L_{2} \subseteq \cdots$ 是 $S$ 中元素的任一个升链, 其中 $\subseteq$ 是集合包含关系, 那末易知 $U L_{i}$ 是 $R$ 的诣零左理想且含 $L$. 因 此, $U L_{i} \in S$, 故由 Zorn 引理, 在 $S$ 中必有一个极大元包含 $L$. 这个极大元即是我们引理结 论所要求的.

定理 1.1. 设 $R$ 是环, $N^{\prime}$ 是 $R$ 的诣零根, $L$ 是 $R$ 的一个诣零左理想. 记 $A=L+L R+N^{\prime}$,

1) 这里所指通常左零化子极大条件：对每一左零化子升链 ${ }^{1} S_{1} \subseteq{ }^{1} S_{2} \subseteq \cdots$, 其中 $S_{i}$ 是子集，必在有限处终止. 
$M=A-N^{\prime}$. 那末 $R$ 满足 $M$ 上 $R$-左模同态链归纳条件, 当且仅当 $\alpha$ 是诣零理想.

证. 充分性: 若 $A$ 是诣零理想, 则 $A \subseteq N^{\prime}$, 因此 $M=\phi . R$ 自然满足 $M$ 上 $R$-左模同态 链归纳条件.

必要性: 我们只要证明 $A=N^{\prime}$. 如果 $A \neq N^{\prime}$, 则作 $M=A-N^{\prime} \neq \phi$. 首先指出, 对 $M$ 中任一元素 $a,{ }^{\perp} a \neq R$. 事实上, 若 ${ }^{\perp} a=R$, 则 $R a=0$. 记 [a] $=R a+a R+R a R+$ $\boldsymbol{Z} a$ ，其中 $\boldsymbol{Z}$ 是整数环，那末易知 $[a]^{2}=0$. 因此 $[a] \subset N^{\prime}$, 这与 $a \in M$ 相矛盾. 我们说, $M$ 的元素 $a$ 使 ${ }^{\perp} a$ 是关于 $M$ 为极大, 若对于任何元素 $b \in M$ 为有 $\perp_{a} \subseteq \perp^{\perp} b$ 必有 $\perp_{a}=\perp_{b}$. 于 是由定理的假设,集合

$$
S=\left\{\left.x \in M\right|^{\perp_{x}} \text { 关于 } M \text { 为极大 }\right\} \text {. }
$$

非空. 现在来引出矛盾. 我们分二步骤:

\section{I. $S$ 中元素不能皆是幕零的.}

现在用反证法来证此论断. 如果 $S$ 中所有元素皆是幕零的, 那末必可得如下结论：对于 $\boldsymbol{S}$ 中任一元素 $\boldsymbol{x}$ 皆有

$$
x \operatorname{Rx} \subset N^{\prime},
$$

现在来证此论断. 令 $a \in R$, 如果 $x a \in N^{\prime}$, 则有 $x a x \in N^{\prime}$. 因此假设 $x a \in N^{\prime}$. 因 $x \in S$, $x a \in M$, 且 ${ }^{i} x a \neq R$, 因此 $x a \in S$. 又因 $S$ 的元素是幂零的, 因此 $x a$ 是幂零元, 因此 $a x$ 也 是幂零元, 故存在正整数 $n$, 使 $(a x)^{n}=0,(a x)^{n-1} \neq 0$. 如果 $x a x \in N^{\prime}$, 则无需再证. 因此 假设 $x a x \in N^{\prime}$, 与刚才所讨论那样,易知 $x a x \in S$. 于是 $R x$ 与 $R x a x$ 是 $R$-模同构, 且 $x$ 映 到 $x a x$. 令 $r^{\prime}=a(x a)^{n-2}$, 则由 $R$-同构性知, $r^{\prime} x a x=0$, 当且仅当 $r^{\prime} x=0$, 于是得出 $(a x)^{n-1}=0$ 的矛盾. 这就是说, $x a x \in N^{\prime}$. 因为 $a$ 是 $R$ 中任取的元素, 故证得 $x R x \subset N^{\prime}$.

令 $[x]=R x+x R+R x \dot{R}+Z x$. 那末由 $(* *)$ 知, $[x]^{3} \subset N^{\prime}$, 因此 $[x]$ 是诣零理想, $x \in N^{\prime}$, 但 $x \in M=A-N^{\prime}$, 因此得出矛盾. 所以证得 $S$ 中的元素不能皆是幕零的.

II. $S$ 中的元素只能是幂零的.

现在来证此论断. 令 $y \in S, y$ 不是幂零元. 因 $y \in A=L+L R+N^{\prime}$, 所以 $y=$ $\sum_{i=1}^{n} \lambda_{i}+\lambda_{0}$, 其中 $\lambda_{0} \in N^{\prime}, \lambda_{1} \in L, \lambda_{i}=l_{i} \omega_{i}, l_{i} \in L, \omega_{i} \in R, i=2, \cdots, n$. 于是 $y \lambda_{0} \in N^{\prime}$, $y \lambda_{1}, y \lambda_{i}=y l_{i} \omega_{i}=l_{i}^{\prime} \omega_{i}$ 皆是幂零元, $l_{i}^{\prime} \in L, i=2, \cdots, n$. 这是因为 $\omega_{i} l_{i}^{\prime} \in L$ 是幂零元, 所 以 $l_{i}^{\prime} \omega_{i}=y \lambda_{i}$ 也是幕零元.

现在要证 $y \lambda_{i} \in N^{\prime}, i=1, \cdots, n$.

现在来证此论断. 如果有某个 $y \lambda_{i} \in N^{\prime}$, 那末由 $y \lambda_{i} \in A$ 得出 $y \lambda_{i} \in M$. 由前面知, ${ }^{\perp} y \lambda_{i}$ $\neq R$, 并且 $y \in S$, 所以 $y \lambda_{i} \in S$. 令 $x=y \lambda_{i}, r \in R$, 那末 $r x=r y \lambda_{i}=l_{i}^{\prime \prime} \omega_{i}$, 其中 $l_{i}^{\prime \prime} \in L$, $\omega_{i} \in R$, 易知 $r x$ 是幕零元. 于是存在正整数 $n$, 使 $(r x)^{n}=0,(r x)^{n-1} \neq 0$. 如果 $x r x \notin$ $N^{\prime}$, 则与前那样可知, $x r x \in S$. 令 $r^{\prime}=r(x r)^{n-2}$, 则由 $r^{\prime} x r x=0$ 得出 $r^{\prime} x=0$, 这与 $r^{\prime} x$ $=(r x)^{n-1} \neq 0$ 矛盾. 因此 $x r x \in N^{\prime}, x R x \subset N^{\prime}$. 与前面那样又得出主理想 $[x] \subset N^{\prime}$, 这与 $x=y \lambda_{i} \notin N^{\prime}$ 的假设相矛盾，因此对于 $i=1, \cdots, n$ 皆有 $y \lambda_{i} \in N^{\prime}$.

现在回到 $y=\sum_{i=1}^{n} \lambda_{i}+\lambda_{0}$ 的形式. 于是 $y^{2}=\sum_{i=1}^{n} y \lambda_{i}+y \lambda_{0} \in N^{\prime}$, 这与 $y$ 不是淂零元相 矛盾. 因此 $S$ 的元素必须皆是幕零元. 
综合上述 (I) 及 (II) 的互为矛盾的结论, 我们得出 $A=N^{\prime}$. 这就证明了定理的必要性.

定义 1.5. 我们称 $R$ 的一个子集 $M$ 为 Köthe 子集,当且仅当存在一个极大诣零左理想 $L$, 使 $M=\left(L+L R+N^{\prime}\right)-N^{\prime}$.

定义 1.6. 我们说环 $R$ 成立 Köthe 猜测, 当且仅当 $R$ 的任一诣零左理想所生成的理想必是 诣零的.

定理 1.2. 设 $R$ 是环,那末 $R$ 成立 Köthe 猜测, 当且仅当 $R$ 对于每个 Köthe 子集上满足 $R-$ 左模同态链归纳条件.

证. 充分性: 设 $L^{\prime}$ 是任一诣零左理想,由引理 1.1 知, 必存在一个极大诣零左理想 $L$ 包 含 $L^{\prime}$. 于是作 Köthe 子集 $M=\left(L+L R+N^{\prime}\right)-N^{\prime}$, 其中 $N^{\prime}$ 是 $R$ 的诣零根. 由充分条 件假设， $R$ 满足 $M$ 上 $R$-左模同态链归纳条件，因此由定理 1.1 得出， $A=L+L R+N^{\prime}$ 是 诣零的, 故 $L^{\prime}+L^{\prime} R$ 是诣零理想.

必要性: 令 $M$ 是一个 $\mathrm{Köthe}$ 子集, 则由定义 1.1 知, 必存在一个极大谐零左理想 $L$, 使 $M=\left(L+L R+N^{\prime}\right)-N^{\prime}$ 。由定理假设, $L+L R$ 是诣零理想，因此 $L+L R \subseteq N^{\prime}$ ，于 是 $M=\phi . R$ 自然满足 $M$ 上 $R$-左模同态链归纳条件，

定理 1.3. 设 $R$ 是环, $N^{\prime}$ 是 $R$ 的诣零根, 那末 $N^{\prime}$ 包含任何诣零单侧理想, 当且仅当 $R$ 对 每个 Köthe 子集上满足 $R$-左模同态链归纳条件.

证. 必要性是显然的. 现在来证充分性,为此我们只要证明 $N^{\prime}$ 包含任何诣零右理想就可 以了，因为定理 1.2 保证 $N^{\prime}$ 包含任何诣零左理想. 记 $I$ 是 $R$ 的任一诣零右理想， $x$ 是 $I$ 的任 一元素, 显然是幕零元, 我们要证 $x \in N^{\prime}$. 容易明白,左理想 $R x$ 必是诣零的, 因此 $R x \subset N^{\prime}$, 于是 $R x R \subset N^{\prime}$. 令 $L=R x+R x R+Z x$ ，我们要证 $L$ 是诣零左理想. 事实上,若 $x^{n}=0$, 那末易知 $L^{m} \subseteq R x+R x R \subset N^{\prime}$ ，所以对每个元素 $l \in L, l$ 必是幕零元，这就得出 $L \subset N^{\prime}$, 因此 $x \in N^{\prime}, I \subset N^{\prime}$.

推论 1.1. 设 $R$ 是环. 那末 $R$ 成立 Köthe 猜测，当且仅当 $R$ 对每个 Köthe 子集上满足特 殊左零化子极大条件.

证. 由性质 1.1 及定理 1.2 得出.

推论 1.2. 设 $R$ 是环. 那末 $R$ 的诣零根包含任何诣零单侧理想, 当且仅当 $R$ 满足任一个 Köthe 子集上特殊左零化子极大条件.

证. 由性质 1.1 及定理 1.3 可得出.

注意: 若 $R$ 满足左零化子极大条件,那末 $R$ 也可以有上述推论的相应结论,但左零化子极 大条件太强无需细述.

本节讨论一个诣零单侧理想在什么条件下是幕零的问题. 在这方面, Herstein 有过这样 猜测：设 $R$ 是满足左零化子极大条件的环，那末 $R$ 的任一诣零单侧理想必是幕零的. 这个猜 测已被否定，最近谢邦杰用左零因子理想条件代替左零化子极大条件证明了 Herstein 的结论 是正确的. 本节将给出 $R$ 的任一诣零单侧理想必是幂零的一个充要条件. 谢邦杰的定理是我 们的一个自然推论.

引理 2.1. 设 $R$ 是一个环, $N^{\prime}$ 是 $R$ 的诣零根, $N$ 是 $R$ 的所有幕零理想之和, 记 $M=N^{\prime}-$ 
$N$. 如果 $R$ 满足 $M$ 上 $R$-左模同态链归纳条件, 那末必有元素 $x \in M$, 使 $[x]^{2} \subset N$, 其中 $[x]$ 表示 $x$ 生成的 $R$ 的主理想.

证. 设 $\cdots \subseteq \subseteq^{\perp} m_{i} \subseteq{ }^{1} m_{i+1} \subseteq \cdots$ 是 $M$ 上任一特殊左零化子链,其中足标集合为 $I$. 那末由性 质 1.1 知, 集合 $\left\{R_{m_{i}}\right\}_{i \in I}$ 是 $M$ 上 $R$-左模同态链. 由引理假设知, 必有元素 $m \in M$, 使 $\left\{R m_{i}\right\}_{l}$ 中任一元素 $R m_{\alpha}$ 模同态地映到 $R m$ 上且 $m_{\alpha}$ 映到 $m$. 于是有 ${ }^{{ }^{\prime}} m_{\alpha} \subseteq{ }^{\perp} m$. 现在要证链 $\left\{{ }^{\perp} m_{i}\right\}_{i \in I}$ 是归纳的. 事实上,若另有元素 $x \in M$, 使 ${ }^{{ }^{\prime}} m_{i} \subseteq \subseteq^{{ }^{\perp}}$, 我们只要证 ${ }^{\perp_{x}} \supseteq^{{ }^{\prime} m}$. 但这是显 见的. 因为 ${ }^{{ }^{2}} m_{i} \subseteq{ }^{\perp} x$, 所以必有模同态 $\varphi_{i}$ 使 $R m_{i}$ 映到 $R x$ 上,且 $m_{i} \varphi_{i}=x$. 于是由引理 的假设及定义 1.3 知, 必有模同态 $\varphi$, 使 $R m$ 映到 $R x$ 上,且 $m \varphi=x$. 这就证得 ${ }^{{ }} m \subseteq^{+} x$. 因此任何链 $\left\{{ }^{\perp} m_{i}\right\}_{i \in I}$ 皆是通常意义下归纳的. 根据 Zorn 引理, 在 $M$ 中必有元素 $m$ 使 ${ }^{{ }^{\prime}} m$ 关 于 $M$ 为极大. 另一方面,对任何 $m_{i} \in M$ 皆有 ${ }^{{ }} m_{i} \neq R$. 不然的话就有主理想 $\left[m_{i}\right]$ 是幂零 理想,于是得出 $\left[m_{i}\right] \subset N$ 的矛盾.

现在来证引理的结论, 自然假设 $M$ 非空. 那末在 $M$ 中必有元素 $m$, 使 ${ }^{{ }} m$ 关于 $M$ 为极 大. 令 $r \in R$, 我们要证 $m r m \in N$. 事实上, 由 $m r \in N^{\prime}$ 得出 $r m$ 必是幕零元, 因此存在正 整数 $n$, 使 $(r m)^{n}=0,(r m)^{n-1} \neq 0$. 如果 $m r m \in M$, 那末 $R m$ 与 $R m r m$ 必是 $R$-模同 构, 且 $m$ 与 $m r m$ 对应. 令 $r^{\prime}=r(m r)^{n-2}$, 那末由 $r^{\prime} m r m=(r m)^{n}=0$, 必得 $r^{\prime} m=0$, 因此 $(r m)^{n-1}=0$, 这与前面 $(r m)^{n-1} \neq 0$ 相矛盾. 因此 $m r m \notin M, m r m \in N$. 但 $r$ 是 $R$ 中任意元, 故有 $m R m \subset N$. 其次, 因为 ${ }^{\perp} m$ 是关于 $M$ 为极大, 所以与上面那样可证 $m^{2} \in N$, 记 $[m]=R m+m R+R m R+Z m$ 是主理想, 那末易知 $[m]^{2} \subset N$.

定理 2.1. 设 $R$ 是环, $N^{\prime}$ 是 $R$ 的诣零根, $N$ 是 $R$ 的所有幕零理想之和, $M=N^{\prime}-N$. 如 果 $R$ 满足 $M$ 上 $R$-左模同态链归纳条件，且 $N$ 是 $R$ 的半素理想，那末 $N=N^{\prime}$.

证. 如果 $M \neq \phi$, 那末由引理 2.1 知, 必有元素 $m \in M,[m]^{2} \subset N$. 由 $N$ 的半素理想性 质得出, $[m] \subset N$, 这与 $m \in M$ 相矛盾, 因此 $N^{\prime}=N$.

定理 2.2. 设 $R$ 是环, $N^{\prime}$ 是诣零根, $N$ 是 $R$ 的所有幕零理想之和, 记 $M=N^{\prime}-N$, 那末 $R$ 的每个诣零单侧理想是幕零的，当且仅当 $R$ 满足 $M$ 上以及每个 Köthe 子集上 $R$-左模同态 链归纳条件并且 $N$ 是幕零的.

证. 必要性: 因为假设每个诣零单侧理想是幂零的, 因此 $N^{\prime}$ 是幂零的,故 $M=N^{\prime}-N$ $=\phi . R$ 自然满足 $M$ 上 $R$-左模同态链归纳条件. 并且 $N$ 是幂零的. 另一方面, 令 $L$ 是任一诣 零左理想,由假设必有 $n$, 使 $L^{n}=0$, 因此 $(L+L R)^{n}=0$. 故任何 Köthe 子集是空集,所 以 $R$ 满足任一Kōthe 上 $R$-左模同态链归纳条件.

充分性: 由定理 2.1 知, $N=N^{\prime}$. 由定理 1.3 知, $N^{\prime}$ 包含任何诣零单侧理想, 因为假设 $N$ 是幕零的, 所以每个诣零单侧理想是幕零.

定理 2.3. 设 $R$ 是环. $N^{\prime}$ 是诣零根, $N$ 是 $R$ 的所有幕零理想之和, 记 $M=N^{\prime}-N$, 那 末 $R$ 的每个诣零单侧理想是幂零, 当且仅当 $R$ 满足 $M$ 上及每个 Köthe 子集上特殊左零化子 极大条件, 并且 $N$ 是幂零的.

证. 由性质 1.1 及定理 2.2 中得出.

推论 2.1. 设 $R$ 是环, $N$ 是 $R$ 的所有幂零理想之和. 若 $R$ 满足左零化子极大条件, 且 $N$ 是 幂零的,那末 $R$ 的任一诣零单侧理想必幂零.

证. 我们的假设满足定理 2.3 的充分条件,因此立刻可从定理 2.3 得出所要求的炶论. 
从推论 2.1 可见, Herstein 猜测之所以被否定是因为设有假设 $N$ 是幂零的缘故.

我们说, $R$ 的左理想 $L$ 是左零因子理想,根据谢邦杰的定义,若在 $R$ 中存在非零子集 $S$, 使 $L S=0$.

推论 2.2 (谢定理)。设环 $R$ 满足左零因子理想极大条件，也就是任何左零因子理想升链 必在有限处终止,那末 $R$ 的任一诣零单侧理想必是幂零的.

证. 由假设得出 $R$ 的所有幕零理想之和 $N$ 必是幂零的, 又因为每个特殊左零化子升链即 是左零因子理想升链，因此定理 2.3 的充分条件满足，故证得 $R$ 的每个诣零单侧理想是幂零 的.

注意：从定理 2.3 结论可看出, 谢邦杰的左零因子理想极大条件还太强, 它不能成为必要 条件，我们不妨用下面例子来说明此点. 并以此例验证定理 2.3 .

例. 设 $R$ 是阿贝尔 (加法)群, $\boldsymbol{Z}$ 是整数环,我们总可假设 $R$ 不满足加法子群升链条件(例 如取 $R$ 为任一无限维问量空间，那末自然不满足加法子群升链条件). 对于 $R$ 我们引进平凡乘 积,即对 $R$ 中任二元素 $a, b$ 定义 $a b=0$, 于是 $R$ 成为一个环, $R^{2}=0$. 记 $S=Z \times R$. 我 们用熟知方法引进加法及乘法如下:

$$
\begin{aligned}
& (m, a)+(n, b)=(m+n, a+b), \\
& (0,0)=0, \\
& (m, a)(n, b)=(m n, n a+m b+a b),
\end{aligned}
$$

其中 $m, n \in \boldsymbol{Z}, a, b \in R$. 于是 $S$ 成为一个环. $(0, R)$ 是 $S$ 的一个理想, 并且 $(0, R)$ 与 $R$ 是 环同构，记 $N^{\prime}$ 是 $S$ 的诣零根, $N$ 是 $S$ 的所有冥零理想之和, 容易知道 $N^{\prime}=N=(0, R)$. 于 是 $M=N^{\prime}-N=\phi$. 任何 Köthe 子集也是空集, 且 $N^{2}=0$, 因此定理 2.3 的充分条件满 足, $S$ 的每个诣零单侧理想必是幂零的,然而推论 2.2 的左零因子理想极大条件并不满足, 因 为 $R$ 的每个加法子群是 $R$ 的左零因子理想, 而由 $R$ 的假设知, 它不满足升链条件,但 $(0, R)$ 与 $R$ 环同构, 因此 $S$ 不满足左零因子理想极大条件.

在结束本节之前, 我们还要对满足左理想极大 (或极小) 条件的环的诣零根必是幕零的经 典定理给出推广形式,并直接给出一个非常简单的证明，我们先给如下定义:

定义 2.1. 我们说环 $R$ 满足诣零左理想极大条件, 若对 $R$ 的任一诣零左理想升链 $L_{1} \subseteq L_{2} \subseteq$ …必有正整数 $n$ 使 $L_{n}=L_{n+1}=\cdots$. 相应地有诣零左理想极小条件的概念.

定理 2.4. 设 $R$ 满足诣零左理想极大条件,那末 $R$ 的诣零根必是幕零的.

证. 由定理假设必可得如下结论： $R$ 的所有幕零理想之和 $N$ 必是幕零的. 记 $N^{\prime}$ 是诣零 根, $M=N^{\prime}-N$. 令 $N_{\perp m}^{\prime}=\left\{r \in N^{\prime} \mid r m=0\right\}, m \in M$ 。 显然, $N_{\perp m}^{\prime}$ 是 $R$ 的一个诣零左理 想, 如果 $M \neq \phi$, 我们要引出矛盾. 首先注意: 对任何元 $m$ 必使 ${ }^{N_{\perp m}^{\prime}} \neq 0$. 如果不然, ${ }_{\perp m}^{\prime}$ $=0$, 则因 $m \in N^{\prime}$, 所以有 $[m]^{2}=0$, 因此 $m \in N$ 矛盾。由假设知, 在 $M$ 中必有元素 $m$, 使 $N_{\perp m}^{\prime}$ 关于 $M$ 为极大. 令 $t \in R$, 要证 $m t m \in N$. 事实上,因 $t m \in N^{\prime}$, 所以必有正整数 $n$, 使 $(\mathrm{tm})^{n}=0,(\mathrm{tm})^{n-1} \neq 0$. 由 ${ }^{N_{\perp m}^{\prime}}$ 的极大元性质知, ${ }^{N_{\perp m}^{\prime}}={ }_{\perp m t m}^{\prime}$. 于是从 $t(m t)^{n-2}$ $=m t m=(t m)^{n}=0$ 得出 $t(m t)^{n-2} m=(t m)^{n-1}=0$ 的矛盾. 因此 $m t m \in N$, 故 $m R m \subset N$. 易知 $[m]^{3} \subset N, m \in N$. 但这与 $m \in M$ 相矛盾,故必须有 $M=\phi$, 即 $N^{\prime}=N$.

定理 2.5. 设 $R$ 是满足诣零左理想极小条件的环, 则 $R$ 的任一诣零单侧理想必是幂零的. 证. 记 $L$ 是 $R$ 的任一诣零左理想. 如果 $L$ 不是幂零, 则由定理假设知, 必有一个诣零极小 
左理想 $L^{\prime} \subseteq L$, 并且 $L^{\prime}$ 不足幂零的, 因此 $L^{\prime 2}=L^{\prime}$. 记 $T$ 是所有使 $L^{\prime} I \neq 0$ 的 $R$ 的诣零 左理想 $I$ 的全体。因 $L^{\prime} \in T$, 所以 $T \neq \phi$. 于是由定理假设, 必有一个极小元 $I_{1} \in T$, 使 $L^{\prime} I_{1} \neq 0$. 因此必有元素 $e_{1} \in I_{1}$, 使 $L^{\prime} e_{1} \neq 0$. 由 $L^{\prime} L^{\prime} e_{1}=L^{\prime} e_{1}$ 及 $L^{\prime} e_{1} \subseteq I_{1}$, 得出 $L^{\prime} e_{1}=$ $I_{1}$, 因此有 $e^{\prime} \in L^{\prime}$, 使 $e^{\prime} e_{1}=e_{1}$. 但 $e^{\prime}$ 是幂零元, 故得出 $e_{1}=0$, 这与 $L^{\prime} e_{1} \neq 0$ 矛盾. 故 $L$ 必是幕零的. 于是由此易证, $R$ 的任一诣零右理想必是幕零的.

\section{考文解}

[1] Herstein, 1, N. \& Small, L. W., Canadian, J. Math., 16 (1964), 771-776.

[2] 谢邦杰,数学进展, 2 (1981)，15̣3-154。 